\title{
NEW DETECTION OF X-RAY PULSAR NEBULAE BY ASCA
}

\author{
N. KAWAI \\ The Institute of Physical and Chemical Research (RIKEN) \\ 2-1 Hirosawa, Wako, Saitama 351-01, Japan \\ KEISUKE TAMURA \\ Venture Business Laboratory, Nagoya University, \\ Chikusa-ku, Nagoya 464-01, Japan
}

AND

S. SHIBATA

Department of Physics, Yamagata University, 1-4-12 Kojirokawa, Yamagata 990, Japan

\section{Observations}

X-ray images of rotation-powered pulsars were examined using ASCA Gas Imaging Spectrometer (GIS). The data sets are taken from those available in the ASCA public archive in the performance verification (PV) phase and the guest-observing (GO) phase 1 . We detected diffuse X-ray sources in the vicinity of nine pulsars including five new detections. There are large variety in their morphology and spatial size. The high probability of finding such diffuse sources around pulsars suggests that they exist universally for all the active pulsars, and that they are powered by the pulsars. We propose that the pulsar-powered nebula is a good probe to measure the otherwise invisible energy flux dissipating from a pulsar into the surrounding space.

\section{Energy Spectra and Luminosity}

The spectra of these nebulae are generally hard, and characterized by the power-law photon index of $1.5 \sim 2.0$. The X-ray luminosities of these pulsar nebulae are derived based on the best-fit power-law models. The fraction of their luminosities in the total rotation energy losses range from $10^{-4}$ to $10^{-2}$. These luminosities are plotted against the spin-down power of the pulsars $\dot{E}_{\text {rot }}(=I \Omega \dot{\Omega})$ in Fig 1 . 


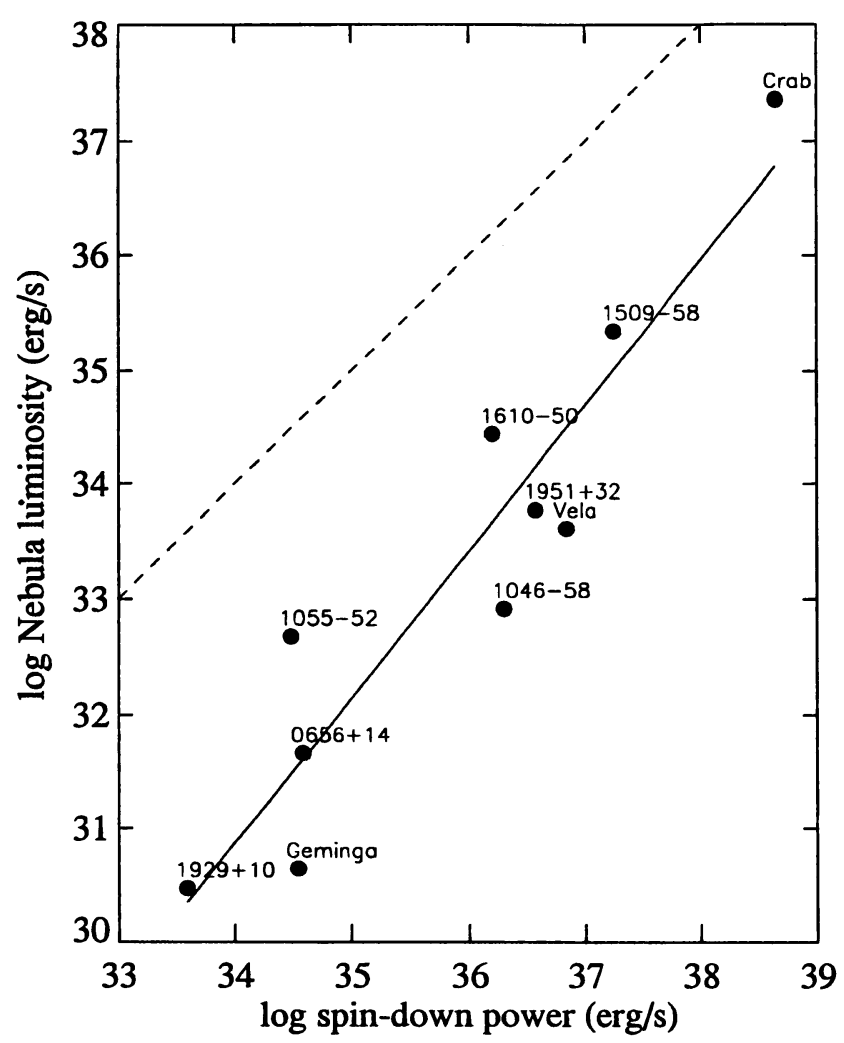

Figure 1. The X-ray pulsar nebula luminosity in the $0.7-10 \mathrm{keV}$ band $L_{\text {nebula }}(0.7-10$ $\mathrm{keV}$ ) plotted as a function of the spin-down power of the pulsars $\dot{E}_{\text {rot }}(=I \Omega \dot{\Omega})$. The empirical relation obtained with least-square fit is shown by a solid line. The dashed line indicates $L_{\text {nebula }}=\dot{E}_{\text {rot }}$.

The least-square fit in the logarithmic space of the ten points here gives an empirical relation:

$$
\log L_{\text {nebula }}=(33.42 \pm 0.20)+(1.27 \pm 0.17) \cdot \log \left(\frac{\dot{E}_{r o t}}{10^{36}}\right)
$$

Here the unit of the power and the luminosity is ergs, and the 1- $\sigma$ uncertainties of the parameters shown in the formula reflect the scatter of the data points rather than the statistical uncertainties of the estimated luminosities. The theoretical interpretation of this relation is given in a separate paper. 